\title{
EURADH 2012 endet mit positivem Fazit
}

Vom 16. bis zum 20. September 2012 fand im Graf-Zeppelin-Haus in Friedrichshafen die von der DECHEMA veranstaltete 9. European Adhesion Conference - EURADH statt. Die behandelten Themen reichten von den Grundlagen der Adhäsion bis hin zu industriellen Aspekten, wobei eine der beiden Parallelsessions jeweils der Mechanik und dem Alterungsverhalten von Klebverbindungen gewidmet war.

\le präsentierten Vorträge zeichneA ten sich durch ein erfreulich hohes wissenschaftliches Niveau aus und regten zu intensiven Diskussionen an. Auch die gezeigten circa 60 Poster waren während der Postersessions stets dicht umlagert und es war für die Jury extrem schwer, die drei Preisträger für die ausgeschriebenen Preise zu ermitteln. Diese gingen an Y. Kawano von der Yokohama National University (Application of epoxy resins of steam explosion lignin derived from softwood), L. Krogh von der Universität des Saarlandes (Determination of viscoelastic properties in adhesive joints) und M. Alfano, King Abdullah University (Identification of a cohesive zone model for debonding of adhesive joints).

Die EURADH war mit 175 Teilnehmern aus 17 Ländern gut besucht, wobei Japan nach Deutschland und Frankreich

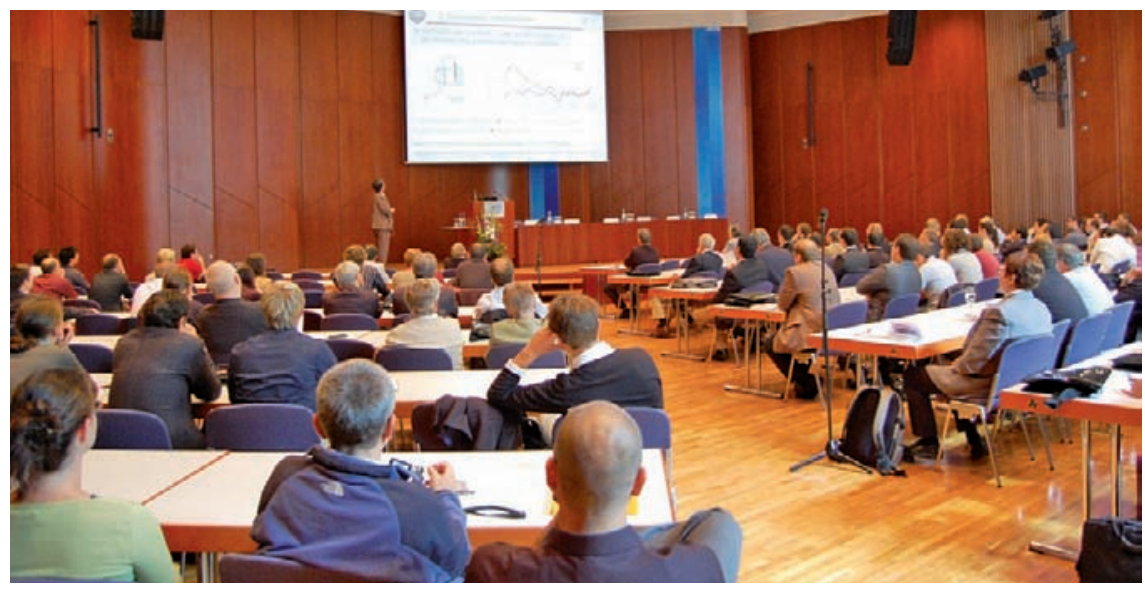

Alle Vorträge zeichneten sich durch ein hohes wissenschaftliches Niveau aus und waren immer gut besucht.

die drittstärkste Delegation stellten. Dies ist sicher auch der mit der EURADH verbundenen nächsten Weltkonferenz WCARP (weitere Infos: www.adhesion.or.jp/wcarp) geschuldet, welche vom 7. bis 10.9.2014 in Nara, Japan, stattfinden wird. Auch die nächste EURADH ist bereits geplant und wird vom 22. bis 25.4.2014 in Alicante, Spanien, stattfinden.

Für die diesjährige Veranstaltung lässt sich insgesamt ein durchweg positives Fazit ziehen. Zwischen den Teilnehmern aus Industrie und Instituten, den verschiedenen Ländern und Fachgebieten gab es einen sehr intensiven Austausch, das Kongresszentrum und seine Umgebung boten ein hervorragendes
Ambiente und schlussendlich rundeten Sonnenschein und eine gute Verpflegung die 9. EURADH ab.

Im Kreis der EURADH-Länder hat sich in der letzten Zeit einiges getan. Traditionell wechselte der Konferenzort zwischen Frankreich, Großbritannien und Deutschland. Nun ist bereits 2010 Spanien als viertes Land mit eigener Adhesion Society hinzugekommen und während der EURADH 2012 wurde die portugiesische Adhesion Society als fünfte Trägerorganisation aufgenommen. Da über die beiden iberischen Länder auch starke Beziehungen nach Südamerika bestehen, verspricht dies für zukünftige Veranstaltungen ein noch breiteres Teilnehmerfeld.

Dr. Andreas Hartwig

\section{BASF erwirbt TDI-Geschäftsanteile von Ciech}

\section{B ASF übernimmt Teile des weltweiten TDI-Geschäfts} (Toluoldiisocyanat) von Ciech - vorausgesetzt, die zuständigen Kartellbehörden stimmen zu. Mit dem Abschluss der Transaktion wird im ersten Quartal 2013 gerechnet.

Die Produktionsanlage der Ciech-Tochtergesellschaft Zachem in Polen ist von der Transaktion nicht betroffen. Ciech und BASF werden eng zusammenarbeiten, um den reibungslosen Transfer von Liefervereinbarungen und Serviceleistungen für Ciechs TDI-Kunden zu gewährleisten.

„Mit dem Erwerb dieses Geschäfts unterstreichen wir unser starkes Engagement in den TDI-Märkten in der Region Eu- ropa, Naher Osten und Afrika sowie weltweit“, sagte Raimar Jahn, Leiter des Unternehmensbereichs Polyurethanes der BASF. „Wir freuen uns auf eine erfolgreiche Zusammenarbeit mit Ciechs Kunden.“ Das deutliche Bekenntnis der BASF zum TDI-Geschäft zeigt auch der Bau der weltgrößten einsträngigen TDI-Anlage am Standort Ludwigshafen.

Dariusz Krawczyk, Vorsitzender des Vorstands von Ciech, fügte hinzu: "Wir sind überzeugt, mit BASF einen idealen Käufer für unser TDI-Geschäft gefunden zu haben. Unsere Kunden werden von der großen Expertise der BASF auf dem Polyurethan-Markt sowie ihrer weltweiten Präsenz profitieren." 